\title{
First-principles theory of frozen-ion flexoelectricity
}

\author{
Jiawang Hong* and David Vanderbilt \\ Department of Physics and Astronomy, Rutgers University, Piscataway, NJ 08854-8019, USA
}

(Dated: November 15, 2018)

\begin{abstract}
We demonstrate that the frozen-ion contribution to the flexoelectric coefficient is given solely in terms of the sum of third moments of the charge density distortions induced by atomic displacements, even for ferroelectric or piezoelectric materials. We introduce several practical supercell-based methods for calculating these coefficients from first principles, and demonstrate them by computing the coefficients for $\mathrm{C}$, Si, $\mathrm{MgO}, \mathrm{NaCl}, \mathrm{SrTiO}_{3}, \mathrm{BaTiO}_{3}$, and $\mathrm{PbTiO}_{3}$. Three important subtleties associated with pseudopotentials, the treatment of surfaces, and the calculation of transverse components are also discussed.

PACS numbers: 77.65.-j,77.90.+k
\end{abstract}

Flexoelectricity $(\mathrm{FxE})$ refers to the linear response of electric polarization to an applied strain gradient [1]. Because a strain gradient breaks inversion symmetry, FxE is always symmetry-allowed, unlike piezoelectricity which arises only in noncentrosymmetric materials. The FxE effect is normally negligible on conventional length scales, but it may become very strong at the nanoscale, where huge strain gradients can significantly affect the functional properties of dielectric thin films, superlattices, and nanostructures. The possibility of large effects at the nanoscale with application to functional devices has caused a recent explosion of experimental interest in flexoelectricity [2-8].

There have been remarkably few theoretical studies of FxE, the main difficulty being that strain gradients are inconsistent with translational symmetry. A classical phenomenological theory focused on lattice-mediated contributions was proposed by Tagantsev $[9,10]$ and later applied to study FxE properties of dielectrics by Maranganti and Sharma [11]. A first attempt at a firstprinciples calculation of FxE is due to Hong et al. [12]. Recently, Resta [13] developed a first-principles theory of FxE that was, however, limited to simple elemental insulators such as $\mathrm{Si}$, and was not implemented in practice. Thus, unlike piezoelectricity, which is routinely calculated using modern first-principles methods in a mature theoretical framework, the theory of FxE remains in a primitive state.

In this Letter, we present a complete theory of the frozen-ion contributions to the FxE coefficient (FEC), which were not addressed in Refs. [9-11]. Working under mixed electric boundary conditions to be defined shortly, we demonstrate that the contribution of a given atom to the frozen-ion FEC is just proportional to the third moment of the change in charge density induced by its displacement. This is true for all insulating crystals, from elemental dielectrics to piezoelectrics and ferroelectrics. Furthermore, we propose several practical supercell-based methods for extracting the FEC from $a b$ initio calculations, show that these give consistent results, and discuss their relative advantages. We re- port the frozen-ion FECs for C, $\mathrm{Si}, \mathrm{MgO}, \mathrm{NaCl}, \mathrm{SrTiO}_{3}$, $\mathrm{BaTiO}_{3}$, and $\mathrm{PbTiO}_{3}$, and discuss the trends that emerge from this data. Finally, we briefly discuss three important subtleties: (i) the issue of pseudopotential dependence; (ii) the question of "surface contributions" to the FxE; and (iii) the treatment of transverse components using current-density response. The extension beyond the frozen-ion case, taking into account the internal lattice relaxations in response to strains and strain gradients, will be reported elsewhere.

Theory.- Our approach here is essentially a generalization of the analysis introduced by Resta [13]. We consider an insulating crystal, fully relaxed at zero electric field $\mathbf{E}$, and oriented such that one of its primitive reciprocal lattice vectors lies along $\hat{x}$. We then identify one entire plane of atoms, corresponding to atom $i$ in the home unit cell and its periodic images normal to $\hat{x}$, and displace the entire plane rigidly by $u_{0 i \beta}$ in direction $\beta$. This is done under electric boundary conditions in which the macroscopic $\mathbf{E}$ continues to vanish away from the displaced plane. In general this induces a step in the macroscopic electrostatic potential, so that if done simultaneously to every $N$ 'th plane of type $i$ along $\hat{x}$, it results in an average $E_{x} \neq 0$; instead what remains unchanged is the electric displacement field $D_{x}$. For this reason, we work at "mixed electric boundary conditions" (MEBC) in which we keep the macroscopic (i.e., supercell-averaged) fields fixed to $E_{y}=E_{z}=0$ and $D_{x}=4 \pi P_{\mathrm{s}, x}$, where $\mathbf{P}_{\mathrm{s}}$ is the spontaneous polarization of the undeformed crystal.

We define the planar-averaged change of charge density induced by this displacement to be

$$
f_{i \beta}(x)=\frac{\partial \bar{\rho}\left(\tau_{i x}+x\right)}{\partial u_{0 i \beta}},
$$

where $\bar{\rho}(x)$ is the $y$ - $z$ planar average of $\rho(\mathbf{r})$ and $\boldsymbol{\tau}_{i}$ is the location of atom $i$ in the unit cell. We also define the moments of the induced charge redistribution via

$$
Q_{i \beta}^{(n, \hat{x})}=A \int d x f_{i \beta}(x) x^{n},
$$

where $A$ is the cell area normal to $\hat{x}$. Note that the zeroth moment $Q_{i \beta}^{(0, \hat{x})}$ vanishes due to charge conservation, 
and that $Q_{i \beta}^{(1, \hat{x})}$ can be identified as the "Callen" or "longitudinal" dynamical charge.

By definition the frozen-ion FEC describes the $\mathbf{P}$ induced by a homogeneous strain gradient $\nu$, that is,

$$
u_{l i x}=\frac{1}{2} \nu\left(l a+\tau_{i x}\right)^{2}
$$

where $l$ is a cell index and $a$ is the lattice constant along $x$. In the spirit of Martin [15] and Resta [13], we approach this state via the long-wave $(q \rightarrow 0)$ limit of a displacement wave $u_{l i \beta}=u_{i \beta} e^{i q\left(l a+\tau_{i x}\right)}$, where $u_{i \beta}=u_{\beta}$ (independent of $i$ ) is small enough that a linear-response approach is appropriate. Then the charge density induced by the displacement of sublattice $i$ is

$$
\bar{\rho}_{i \beta}(x)=u_{i \beta} \sum_{l} e^{i q\left(l a+\tau_{i x}\right)} f_{i \beta}\left(x-l a-\tau_{i x}\right) .
$$

This has Fourier components at $\bar{\rho}_{i \beta}(q+G)$ at all $G=$ $2 \pi m / a$, but we focus on the $G=0$ component defined by $\bar{\rho}_{i \beta}(q)=(1 / a) \int_{0}^{a} d x e^{-i q x} \bar{\rho}_{i \beta}(x)$ and obtain

$$
\begin{aligned}
\bar{\rho}_{i \beta}(q) & =\frac{u_{i \beta}}{a} \int_{-\infty}^{\infty} d x^{\prime} e^{-i q x^{\prime}} f_{i \beta}\left(x^{\prime}\right) \\
& =\frac{u_{i \beta}}{V}\left(-i q Q_{i \beta}^{(1, \hat{x})}-\frac{q^{2}}{2} Q_{i \beta}^{(2, \hat{x})}+i \frac{q^{3}}{6} Q_{i \beta}^{(3, \hat{x})}\right)
\end{aligned}
$$

where $x^{\prime}=x-l a-\tau_{i x}$ is used to obtain the first line and the series expansion of $e^{-i q x}$ is used to obtain the second (terms of order $q^{4}$ and higher have been dropped), and $V=a A$ is the cell volume [14]. Restoring $u_{i \beta}=u_{\beta}$ we get a total $\bar{\rho}_{\beta}(q)=\sum_{i} \bar{\rho}_{i \beta}(q)$, and using Poisson's equation in the form $\bar{\rho}(q)=-i q P_{x}(q)$, this implies a polarization modulation

$$
P_{x, \beta}(q)=\frac{u_{\beta}}{V}\left(-i \frac{q}{2} Q_{\beta}^{(2, \hat{x})}-\frac{q^{2}}{6} Q_{\beta}^{(3, \hat{x})}\right)
$$

where $Q_{\beta}^{(2, \hat{x})}=\sum_{i} Q_{i \beta}^{(2, \hat{x})}$ and $Q_{\beta}^{(3, \hat{x})}=\sum_{i} Q_{i \beta}^{(3, \hat{x})}$. The first term of Eq. (5) has dropped out due to the acoustic sum rule $\sum_{i} Q_{i \beta}^{(1, \hat{x})}=0$.

Now we define the (unsymmetrized) strain tensor and gradient of the strain tensor to be, respectively,

$$
\eta_{\beta \gamma}(\mathbf{r})=\frac{\partial u_{\beta}(\mathbf{r})}{\partial r_{\gamma}}, \quad \nu_{\beta \gamma \delta}(\mathbf{r})=\frac{\partial \eta_{\beta \gamma}(\mathbf{r})}{\partial r_{\delta}} .
$$

For the wave $u_{\beta}(\mathbf{r})=u_{\beta} e^{i q x}$ this implies $\eta_{\beta x}(q)=i q u_{\beta}$ and $\nu_{\beta x x}(q)=-q^{2} u_{\beta}$, with other elements such as $\eta_{\beta y}$ vanishing. We also define the (unsymmetrized) frozenion piezoelectric and FxE coefficients to be

$$
e_{\alpha \beta \gamma}=\frac{\partial P_{\alpha}}{\partial \eta_{\beta \gamma}}, \quad \mu_{\alpha \beta \gamma \delta}=\frac{\partial P_{\alpha}}{\partial \nu_{\beta \gamma \delta}},
$$

which we interpret in the spirit of the long-wave method as $e_{\alpha \beta \gamma}=\lim _{q \rightarrow 0} \partial P_{\alpha}(q) / \partial \eta_{\beta \gamma}(q)$ etc. Combining the above expressions with Eq. (6), it follows that [14]

$$
e_{x \beta x}=-\frac{1}{2 V} Q_{\beta}^{(2, \hat{x})},
$$

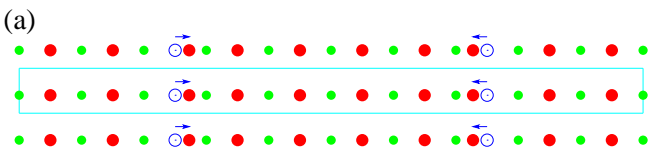

(b)

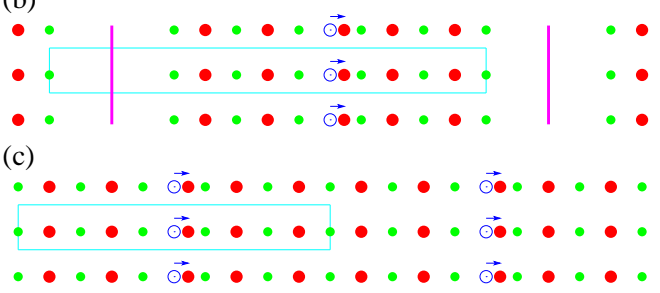

FIG. 1: (Color online) Supercell geometries. Large (red) and small (green) dots are two species of atoms; open dots are atoms before being displaced as shown by arrows. Rectangles indicate supercells. (a) Bulk supercell for Method A. (b) Slab supercell for method B; vertical lines indicate dipole correction layers in vacuum. (c) Bulk supercell for Method C.

$$
\mu_{x \beta x x}=\frac{1}{6 V} Q_{\beta}^{(3, \hat{x})} .
$$

Eq. (9) expresses the frozen-ion (or "purely electronic") piezoelectric tensor in terms of induced quadrupoles quantified by the elements of $Q^{(2, \hat{x})}$. This is basically the same as the result given in the classic paper of Martin [15], except that here all quantities are defined in the MEBC (fixed $D_{x}, E_{y}$, and $E_{z}$ ). Similarly, Eq. (10) corresponds to the induced-octupole formulation derived in Resta's Ref. [13] and agrees with Eq. (22) therein (our $Q^{(3)}$ is Resta's $A Q^{(3)}$ ). Note, however, that Resta's derivation was limited to elemental (and therefore non-polar and non-piezoelectric) crystals. Instead, the derivation here is general, showing that the frozenion $\mathrm{FxE}$ response has contributions only from the induced octupole term.

First-principles calculations.-To compute the FECs from Eq. (10) using ab-initio methods, we need to set up a supercell calculation that allows us to calculate the $f_{i \beta}(x)$ and, from these, the $Q_{i \beta}^{(3, \hat{x})}$, under MEBC $\left(\Delta D_{x}=E_{y}=E_{z}=0\right)$. We have designed three independent procedures to accomplish this, using three different supercell configurations. In Method A, shown in Fig. 1(a), a supercell is built from $N$ repetitions of the bulk cell, and then two atomic layers are displaced in opposite directions under the usual boundary conditions in which the supercell-averaged $\mathbf{E}=0$. Since the induced dipoles are equal and opposite, they compensate each other, $\Delta \mathbf{P}=\Delta \mathbf{D}=0$, and the MEBC are satisfied. In Method B, shown in Fig. 1(b), the supercell contains a slab cut from the bulk material; one central layer is displaced, and there is an external dipole layer in the vacuum that is constantly readjusted so that $E_{x}$ in the vacuum region does not change. Again, as long as there is no free charge on the surfaces, this enforces $\Delta D_{x}=0$. Finally, in Method C, illustrated in Fig. 1(c), the super- 
TABLE I: First and third moments of displacement-induced charge density for $\mathrm{MgO}$ using three different methods.

\begin{tabular}{lrrrrrr}
\hline \hline & \multicolumn{3}{c}{$Q^{(1)}(e)$} & \multicolumn{3}{c}{$Q^{(3)}\left(e \mathrm{Bohr}^{2}\right)$} \\
Method & \multicolumn{1}{c}{$\mathrm{A}$} & \multicolumn{1}{c}{$\mathrm{B}$} & \multicolumn{1}{c}{$\mathrm{C}$} & \multicolumn{1}{c}{$\mathrm{A}$} & $\mathrm{B}$ & \multicolumn{1}{c}{$\mathrm{C}$} \\
\hline $\mathrm{Mg}$ & 0.63 & 0.63 & 0.63 & -8.91 & -8.79 & -8.35 \\
$\mathrm{O}$ & -0.63 & -0.63 & -0.63 & -12.96 & -12.77 & -13.12 \\
Sum & 0.00 & 0.00 & 0.00 & -21.87 & -21.56 & -21.47 \\
\hline \hline
\end{tabular}

cell is again bulk-like, but only one layer is displaced, now using a first-principles code capable of enforcing $\Delta D_{x}=0$. In each case, the supercell size or slab thickness has to be chosen large enough that the induced charge disturbances $f_{i \beta}(x)$ do not overlap or extend to the surface.

The calculations have been performed within densityfunctional theory. We used the local-density approximation [16] for $\mathrm{C}, \mathrm{Si}, \mathrm{MgO}, \mathrm{NaCl}$ and $\mathrm{SrTiO}_{3}$, and the generalized gradient approximation [17] for $\mathrm{BaTiO}_{3}$ and $\mathrm{PbTiO}_{3}$. We used SIESTA [18] for Methods A and B, ABINIT [19, 20] for Method C, and ELK [21] for the all-electron calculations to be discussed later. Supercells were built from 12 unit cells for the perovskites and 8 conventional cells for $\mathrm{C}, \mathrm{Si}, \mathrm{MgO}$ and $\mathrm{NaCl}$ in Method $\mathrm{A}$ and 4 conventional cells for $\mathrm{MgO}$ in Methods $\mathrm{B}$ and $\mathrm{C}$; slabs in $\mathrm{B}$ are separated by $20 \AA$ of vacuum. Atomic displacements of 0.04 Bohr were used in SIESTA and ABINIT, and 0.015 Bohr in ELK.

Table I shows the first and third moments of $\mathrm{MgO}$ $\left(Q^{(2)}=0\right.$ by symmetry) from Methods A-C using identical norm-conserving pseudopotentials. Clearly the results are in good agreement, confirming the consistent implementation of MEBC in all three approaches. Methods A and B can be used to calculate FECs using standard first-principles electronic-structure codes (although Method B requires a vacuum-dipole capability), but they require larger supercells. Converged results can be obtained using smaller supercells with Method C, but only using a code that implements fixed- $D$ electric boundary conditions [20].

Table II lists the moments and FECs for several materials. For elemental and binary dielectrics, it shows that $\left|\mu_{x x x x}\right|$ decreases as ionicity increases. While the anion $\left|Q^{(3)}\right|$ increases from $\mathrm{MgO}$ to $\mathrm{NaCl}$, the cation contribution decreases, and cell volume effects also play an important role. For all the $\mathrm{ABO}_{3}$ perovskite structures, the frozen-ion FECs are remarkably similar. The largest contribution comes from the A atoms, unlike the (Callen) dynamical charges $Q^{(1)}$, for which Ti and $O_{1}$ give dominant contributions.

Rigid-ion model and pseudopotential dependence.Note that the $Q^{(3)}$ moments reported in Tables I and II, and hence the $\mu_{x x x x}$, are all negative. To see why, consider a model in which each cation or anion is represented by a spherically symmetric charge $\rho_{i}(r)$ that displaces rigidly as a unit. A brief calculation shows that
TABLE II: Lattice constants (of conventional cell [14]; $a$ and $c$ for $\mathrm{FE} \mathrm{PbTiO}_{3}$ ), first and third moments, and FECs as obtained using Method A.

\begin{tabular}{|c|c|c|c|c|c|}
\hline & $\begin{array}{c}a \\
\text { (Bohr) } \\
\end{array}$ & & $\begin{array}{c}Q^{(1)} \\
(e)\end{array}$ & $\begin{array}{c}Q^{(3)} \\
\left(e \mathrm{Bohr}^{2}\right) \\
\end{array}$ & $\begin{array}{c}\mu_{x x x x} \\
(\mathrm{pC} / \mathrm{m})\end{array}$ \\
\hline $\mathrm{C}$ & 6.69 & $\mathrm{C}$ & 0.00 & -13.01 & -175.4 \\
\hline $\mathrm{Si}$ & 10.22 & $\mathrm{Si}$ & 0.00 & -27.94 & -105.7 \\
\hline $\mathrm{MgO}$ & 7.73 & $\begin{array}{l}\mathrm{Mg} \\
\mathrm{O}\end{array}$ & $\begin{array}{r}0.63 \\
-0.63\end{array}$ & $\begin{array}{r}-8.91 \\
-12.96\end{array}$ & -95.6 \\
\hline $\mathrm{NaCl}$ & 10.66 & $\begin{array}{l}\mathrm{Na} \\
\mathrm{Cl}\end{array}$ & $\begin{array}{r}0.45 \\
-0.45\end{array}$ & $\begin{array}{r}-1.18 \\
-27.59\end{array}$ & -47.9 \\
\hline $\mathrm{SrTiO}_{3}$ & 7.31 & $\begin{array}{l}\mathrm{Sr} \\
\mathrm{Ti} \\
\mathrm{O}_{1} \\
\mathrm{O}_{3}\end{array}$ & $\begin{array}{r}0.39 \\
1.20 \\
-0.92 \\
-0.33\end{array}$ & $\begin{array}{r}-54.81 \\
-16.48 \\
-27.53 \\
-6.59\end{array}$ & -144.7 \\
\hline $\mathrm{BaTiO}_{3}$ & 7.52 & $\begin{array}{l}\mathrm{Ba} \\
\mathrm{Ti} \\
\mathrm{O}_{1} \\
\mathrm{O}_{3}\end{array}$ & $\begin{array}{r}0.40 \\
1.11 \\
-0.89 \\
-0.31\end{array}$ & $\begin{array}{r}-65.16 \\
-13.80 \\
-27.10 \\
-6.78\end{array}$ & -141.9 \\
\hline $\mathrm{PbTiO}_{3}$ & 7.43 & $\begin{array}{l}\mathrm{Pb} \\
\mathrm{Ti} \\
\mathrm{O}_{1} \\
\mathrm{O}_{3}\end{array}$ & $\begin{array}{r}0.44 \\
0.83 \\
-0.69 \\
-0.29\end{array}$ & $\begin{array}{r}-59.03 \\
-25.56 \\
-23.09 \\
-9.57\end{array}$ & -156.0 \\
\hline $\begin{array}{l}\mathrm{PbTiO}_{3} \\
\quad(\mathrm{FE})[22]\end{array}$ & $\begin{array}{l}7.35 \\
7.88\end{array}$ & $\begin{array}{l}\mathrm{Pb} \\
\mathrm{Ti} \\
\mathrm{O}_{1} \\
\mathrm{O}_{3}\end{array}$ & $\begin{array}{r}0.51 \\
0.76 \\
-0.65 \\
-0.31\end{array}$ & $\begin{array}{r}-57.40 \\
-28.41 \\
-20.61 \\
-9.60\end{array}$ & -148.9 \\
\hline
\end{tabular}

$Q_{i}^{(3)}=\int d^{3} r x^{3}\left(-\partial_{x} \rho_{i}(r)\right)=4 \pi \int d r r^{4} \rho_{i}(r)$. The positive nuclear charge at $r=0$ makes no contribution, so within this model all $Q_{i}^{(3)}<0$. It is not surprising, then, that the real system shows a similar behavior.

The above analysis also implies that the $Q_{i}^{(3)}$, and hence $\mu_{x x x x}$, should depend on the treatment of the core density and the pseudopotential construction. (By contrast, $Q^{(1)}$, and hence $e_{x x x}$, is unaffected.) For example, if the ion charge density is partitioned into core and valence contributions in the above rigid-ion model, both parts will contribute. We illustrate this in Table III by presenting results for $\mathrm{MgO}$ based on two approaches: an all-electron (AE) calculation, and a pseudopotential (PS) calculation in which only the change in valence electron density is used to define $f_{i \beta}(x)$, as for the results presented in Tables I and II. We confirm that AE and PS results agree for the piezoelectric contributions, but find a significant difference for the FxE ones.

This difference arises as follows. Suppose the cellaveraged electrostatic potentials $\bar{\phi}^{\mathrm{AE}}$ and $\bar{\phi}^{\mathrm{PS}}$ are adjusted such that the valence-band maxima $\varepsilon_{\mathrm{VBM}}$ agree between the two bulk calculations. If the PS is of high quality, other features of the bandstructure, as well as forces etc., will show good agreement. However, $\bar{\phi}^{\mathrm{AE}} \neq \bar{\phi}^{\mathrm{PS}}$ because $-e \phi(\mathbf{r})$ is typically much deeper in the $\mathrm{AE}$ core region. Similarly, strain derivatives will also differ: $d \bar{\phi}^{\mathrm{AE}} / d \eta_{x x} \neq d \bar{\phi}^{\mathrm{PS}} / d \eta_{x x}$. For a strain gradient at fixed $D_{x}$ we have $4 \pi \Delta P_{x}=-\Delta E_{x}=d \bar{\phi} / d x=$ 
TABLE III: Moments of MgO obtained from Method A using all-electron (AE) approach or pseudopotential without (PS) or with $(\mathrm{PS}+)$ rigid-core correction.

\begin{tabular}{lrrrrr}
\hline \hline & $Q^{(1)}(e)$ & \multicolumn{3}{c}{$Q^{(3)}\left(e \mathrm{Bohr}^{2}\right)$} \\
& \multicolumn{1}{c}{$\mathrm{AE}$} & \multicolumn{1}{c}{$\mathrm{PS}$} & $\mathrm{AE}$ & $\mathrm{PS}$ & $\mathrm{PS}+$ \\
\hline $\mathrm{Mg}$ & 0.62 & 0.63 & -14.57 & -8.91 & -13.76 \\
$\mathrm{O}$ & -0.62 & -0.63 & -12.38 & -12.96 & -13.02 \\
Sum & 0.00 & 0.00 & -26.95 & -21.87 & -26.80 \\
\hline \hline
\end{tabular}

$\left(d \bar{\phi} / d \eta_{x x}\right)\left(d \eta_{x x} / d x\right)$ so that $\mu_{x x x x}=\left(d \bar{\phi} / d \eta_{x x}\right) / 4 \pi$. We therefore expect $\mu_{x x x x}^{\mathrm{AE}} \neq \mu_{x x x x}^{\mathrm{PS}}$. Similar considerations apply to the theory of deformation potentials, which also depend on the moments $Q^{(3)}[23,24]$.

The difference between $Q^{(3, \mathrm{AE})}$ and $Q^{(3, \mathrm{PS})}$ is unimportant for some purposes, as for obtaining the spatial gradient of $\varepsilon_{\mathrm{VBM}}$ induced by a strain gradient, where it cancels out of the final result. Otherwise, there is a simple fix: for each atom type, we compute a "rigid core correction" (RCC) $Q_{i}^{(3, \mathrm{RCC})}=4 \pi \int d r r^{4}\left[\rho_{i}^{\mathrm{AE}}(r)-\rho_{i}^{\mathrm{PS}}(r)\right]$ using the densities from free-atom AE and PS calculations, and then add these $Q_{i}^{(3, \mathrm{RCC})}$ corrections to the $Q^{(3, \mathrm{PS})}$ values. We have done this for $\mathrm{Mg}$ and $\mathrm{O}$, obtaining $Q^{(3, \mathrm{RCC})}=-4.85$ and $-0.06 e \mathrm{Bohr}^{2}$ respectively. The corrected values, shown in the last column of Table III, are now in good agreement with the AE ones.

Surface contributions.-We also considered calculating $\mu_{x x x x}$ by constructing a slab supercell with two surfaces, as in Fig. 1(b), but applying layer displacements corresponding to the homogeneous strain gradient of Eq. (3). Letting $p_{x}$ be the total slab (TS) dipole per unit area, we can define a FEC via $\mu_{x x x x}^{\mathrm{TS}}=p_{x} / \nu L$, where $\nu=\nu_{x x x}$ and $L$ is the slab thickness. However, we find that $\mu_{x x x x}^{\mathrm{TS}}$ does not agree with the FEC computed using Methods A-C. On the other hand, if we compute the FEC from the slope of the electrostatic potential in the interior of the slab using window convolutions as in Ref. [13], we obtain $\mu_{x x x x}=-E_{x} / 4 \pi \nu$ in good agreement with the results of Methods A-C. (In comparison with Method B, however, we found this method to be more difficult to implement and slower to converge with slab thickness.)

To explain why $\mu_{x x x x}^{\mathrm{TS}} \neq \mu_{x x x x}$, we note that $\mu_{x x x x}^{\mathrm{TS}}$ contains contributions from the slab surfaces. To see this, write $4 \pi p_{x}=\phi_{\mathrm{R}}^{\mathrm{vac}}-\phi_{\mathrm{L}}^{\mathrm{vac}}=\delta \phi_{\mathrm{R}}-E_{x} L-\delta \phi_{\mathrm{L}}$, where $\mathrm{R}$ and $\mathrm{L}$ are right and left surfaces, and for each surface $\delta \phi=\phi^{\text {vac }}-\bar{\phi}$, the difference between the vacuum level just outside and the macroscopic potential just inside the surface. Dividing by $-4 \pi \nu L$, we find $\mu_{x x x x}^{\mathrm{TS}}=\mu_{x x x x}+$ $\left(\delta \phi_{\mathrm{R}}-\delta \phi_{\mathrm{L}}\right) / 4 \pi \nu L$. Now even if the two surfaces were identical initially, in the presence of the strain gradient $\nu$ they exist at different strain states, $\Delta \eta_{x x}=\nu L$, and thus have different $\delta \phi$ values. In linear response we expect $\delta \phi_{\mathrm{R}}-\delta \phi_{\mathrm{L}}=\Delta \eta_{x x}\left(d \delta \phi / d \eta_{x x}\right)$, from which it follows that $\mu_{x x x x}^{\mathrm{TS}}=\mu_{x x x x}+\left(d \delta \phi / d \eta_{x x}\right) / 4 \pi$. The second term is surface-specific [25] and reflects the dependence of the surface work function on local strain.

Because we prefer that the FEC should be defined as a bulk property independent of surface termination, we adopt $\mu_{x x x x}$, and not $\mu_{x x x x}^{\mathrm{TS}}$, as our definition of the FEC. In a sense, $\mu_{x x x x}$ and $\mu_{x x x x}^{\mathrm{TS}}$ are analogous respectively to the "proper" and "improper" contributions to piezoelectricity $[26]$.

Transverse components.-The derivation of Eqs. (9-10) yielded $e_{\alpha \beta x}$ and $\mu_{\alpha \beta x x}$ only for the case $\alpha=x$. We can remove this restriction by replacing Eq. (1) by

$$
\mathcal{P}_{\alpha, i \beta}(x)=\frac{\partial \bar{J}_{\alpha}\left(\tau_{i x}+x\right)}{\partial \dot{u}_{0 i \beta}}
$$

where $\bar{J}_{\alpha}(x)$ is the $y$ - $z$ planar average of the current density in direction $\alpha$ induced by the adiabatic motion $\dot{u}_{0 i \beta}$ of atomic plane $i$ in direction $\beta$, again under MEBC. Defining moments $J_{\alpha, i \beta}^{(n, \hat{x})}=A \int d x \mathcal{P}_{\alpha, i \beta}(x) x^{n}$, Eq. (6) for the polarization in direction $\alpha$ induced by motions in direction $\beta$ is replaced by

$$
P_{\alpha \beta}(q)=\frac{u_{\beta}}{V}\left(-i q J_{\alpha \beta}^{(1, \hat{x})}-\frac{q^{2}}{2} J_{\alpha \beta}^{(2, \hat{x})}\right)
$$

where $J_{\alpha \beta}^{(n, \hat{x})}=\sum_{i} J_{\alpha, i \beta}^{(n, \hat{x})}$. It follows that

$$
e_{\alpha \beta x}=-\frac{1}{V} J_{\alpha \beta}^{(1, \hat{x})}, \quad \mu_{\alpha \beta x x}=\frac{1}{2 V} J_{\alpha \beta}^{(2, \hat{x})} .
$$

For the longitudinal case $\alpha=x$, this result is equivalent to Eqs. (9-10), since continuity implies $\nabla \cdot \mathcal{P}_{i \beta}(\mathbf{r})=-f_{i \beta}(\mathbf{r})$, from which it follows that $Q_{i \beta}^{(n+1, \hat{x})}=(n+1) J_{x, i \beta}^{(n, \hat{x})}$. By contrast, the moments $J_{\alpha, i \beta}^{(n, \hat{x})}$ for $\alpha \neq x$ contain additional information about the transverse motions (e.g., $J_{y, i \beta}^{(0, \hat{x})}$ are transverse, or Born, charges).

In principle, the $\mathcal{P}_{\alpha, i \beta}(x)$ and their moments $J_{\alpha, i \beta}^{(n, \hat{x})}$ are computable using the methods of density-functional perturbation theory. While we have not implemented such a calculation here, Eq. (13) formally solves the problem of extending the present theory to the tensor elements $e_{\alpha \beta x}$ and $\mu_{\alpha \beta x x}$. By carrying out similar calculations with different crystal axes aligned along $\hat{x}$, it should be possible to obtain the full tensors, although care must be taken to account for the modified interpretation of the MEBC after the crystal is rotated.

Conclusions.-We have shown that the longitudinal frozen-ion FEC is proportional to the third moment of induced charge density under MEBC. An extension using the second moment of the induced current density yields also the transverse FECs. This formulation is exact for all insulating crystals. Furthermore, three practical methods for calculating FECs using ab initio methods have been demonstrated by computing the frozen-ion FECs for several materials. Issues concerning pseudopotential dependence and surface effects have also been discussed. Although it remains to include lattice contributions associated with internal relaxations that can occur 
in response to strains and strain gradients, our work represents an important step in the direction of a full firstprinciples theory of FxE.

This work was supported by ONR grant N00014-05-10054. Computations were done at the Center for Piezoelectrics by Design.

* Electronic address: hongjw10@physics.rutgers.edu

[1] S.M. Kogan, Sov. Phys.-Solid. State 5, 2069 (1964).

[2] W.H. Ma, L.E. Cross, Appl. Phys. Lett. 81, 3440 (2002).

[3] L.E. Cross, J. Mater. Sci. 41, 53 (2006).

[4] W.H. Ma, Phys. Status Solidi b 245, 761 (2008).

[5] G. Catalan, L.J. Sinnamon and J.M. Gregg, J. Phys.: Condens. Matter. 16, 2253 (2004).

[6] G. Catalan, et al., Phys. Rev. B 72, 020102 (2005).

[7] P. Zubko, et al., Phys. Rev. Lett. 99, (2007).

[8] D. Lee, et al., Phys. Rev. Lett. 107, 057602 (2011).

[9] A. K. Tagantsev, Phys. Rev. B 34, 5883 (1986).

[10] A. K. Tagantsev, Phase Transitions 35, 119 (1991).

[11] R. Maranganti and P. Sharma, Phys. Rev. B 80,054109 (2009).
[12] J. Hong, G. Catalan, J. F. Scott, and E. Artacho, J. of Phys.: Condens. Matter. 22, 112201 (2010).

[13] R. Resta, Phys. Rev. Lett. 105, 127601 (2010).

[14] In Eqs. (9-10), $V$ may be either the conventional or primitive cell volume, as long as the sum in $Q_{\beta}^{(n, \hat{x})}=\sum_{i} Q_{i \beta}^{(n, \hat{x})}$ runs over the atoms contained in this volume.

[15] R.M. Martin, Phys. Rev. B 5, 1607 (1972).

[16] J.P. Perdew and A. Zunger, Phys. Rev. B 23, 5048 (1981).

[17] Z. Wu and R. E. Cohen, Phys. Rev. B 73, 235116 (2006).

[18] J. M. Soler, et al., J. Phys.: Condens. Matter. 14, 2745 (2002).

[19] X. Gonze, et al., Cmp. Mat. Sci. 25, 478 (2002).

[20] J.W. Hong and D.Vanderbilt, arXiv:1106.5668v1

[21] http://elk.sourceforge.net/

[22] $Q^{(2)}$ is $0.22,0.01,1.14$ and 0.26 for $\mathrm{Pb}, \mathrm{Ti}, \mathrm{O}_{1}$ and $\mathrm{O}_{3}$, respectively.

[23] R. Resta, L. Columbo, and S. Baroni, Phys. Rev. B 41, 12358 (1990).

[24] R. Resta, Phys. Rev. B 44, 11035 (1991).

[25] While a "surface contribution" appears in Eqs. (12-13) of Ref. [9], our context and our definitions are quite different.

[26] D. Vanderbilt, J. Phys. Chem. Solids 61, 147 (2000). 